\title{
Prediction of Effective Elastic Characteristics of a Multiphase Composite Material with a Spatially Reinforced System of Fibers
}

\author{
E.M. Romanovskaia ${ }^{1, \text { a) }}$, E.A. Mityushov ${ }^{1, \text { b) }}$, S.A.Berestova ${ }^{1, c)}$ \\ ${ }^{1}$ Ural Federal University, Mira 19, Yekaterinburg, Russia, 620002 \\ a)Corresponding author: E.M.Romanovskaia@urfu.ru \\ b)mityushov-e@mail.ru \\ ${ }^{c)}$ s.a.berestova@urfu.ru
}

\begin{abstract}
The paper proposes a method based on the operation of averaging properties at different orientations of the axes of elastic symmetry of the material in the areas, each of which is a unidirectional monotropic material, and obtained the final expression for the coefficients of elasticity and compliance by introducing reinforcement parameters characterizing the location of the fibers in the composite material. The obtained expression for the elastic characteristics of a spatially reinforced multiphase composite material allows, by varying the elastic characteristics of the phase components, the volume concentrations and the distribution of fibers in space, to obtain a material with the required level of properties.

Keywords: composite material, multiphase spatially reinforced composite material, texture parameters, young's modulus, Hooke's law, effective characteristics, anisotropy, fibers, macroscopic modulus of elasticity, orthotropic composite material
\end{abstract}

\section{INTRODUCTION}

Currently, more and more attention is attracted to composite materials with a spatial arrangement of fiber reinforcement, introduced into the material, to determine the level of strength properties of the material. In this case, the stresses arising during loading are perceived mainly by reinforcing fibers, in this regard, it is necessary to assess the strength and physical properties of such systems, as well as the ability to predict the elastic properties of these composite materials by the elastic characteristics of the components. The introduction of a spatial framework increases the bearing capacity of the material in thick-walled structures, especially in areas of concentrated loads, cuts, ribs with non-stationary force and temperature effects characteristic of modern technology.

\section{STATEMENT OF THE PROBLEM AND THE METHOD OF SOLVING IT}

As a model, we take a fibrous material consisting of a matrix and reinforcement fibers of various types. The fibers in the isotropic matrix are oriented chaotically and have isotropic or transversally isotropic properties. Three orthogonal planes of elastic symmetry pass through each point of the material, so the composite is orthotropic and is characterized by nine independent elastic constants. It is necessary to obtain expressions for macroscopic modules of elasticity and malleability of such composite material.

Calculation of elastic characteristics will be carried out in several stages. First, we determine the effective characteristics of the composite consisting of a matrix material and fibers of the first type, then determine the elastic characteristics of the composite consisting of a matrix and fibers of the second type, and so on. Then, given that the composite material under consideration consists of a matrix and inclusions of various types, we determine the elastic characteristics of such material from the expression for the mean stress tensor in a heterogeneous system.

International Conference of Numerical Analysis and Applied Mathematics ICNAAM 2019

AIP Conf. Proc. 2293, 120012-1-120012-4; https://doi.org/10.1063/5.0031297

Published by AIP Publishing. 978-0-7354-4025-8/\$30.00 


\section{THE ELASTIC MODULI OF MULTIPHASE SPATIAL-REINFORCED COMPOSITE}

Elastic modulus and compliance coefficients relate stresses to deformations. Stress $\sigma$ and deformation $\varepsilon$ are second-rank tensors whose components are in a rectangular Cartesian coordinate system $0 \mathrm{x}_{1} \mathrm{x}_{2} \mathrm{x}_{3}$ will be denoted by $\sigma_{\mathrm{ij}}$ and $\varepsilon_{\mathrm{ij}}$. Then, the elastic properties of the material are described by fourth-rank tensors of elastic moduli $\mathrm{c}$ and stress coefficients $\mathrm{s}$ with components $\mathrm{c}_{\mathrm{ijkl}}$ и $\mathrm{s}_{\mathrm{ijkl}}$, respectively. The generalized Hooke's law, which describes the elastic behavior of linearly elastic bodies, can be expressed both in terms of c and s: $\varepsilon=s \sigma, \sigma=c \varepsilon$, either component wise: $\varepsilon_{i j}=s_{i j k l} \sigma_{k l}, \sigma_{i j}=c_{i j k l} s_{k l}$.

The tensors of elastic moduli and stress coefficients are reciprocal.

At the first stage, we determine the elastic macroscopic characteristics of a unidirectional reinforced composite. The second step is the averaging over orientations:

$$
c_{i j k l}=\left\langle l_{i p} l_{j q} l_{k r} l_{l s}\right\rangle c_{p k r s}^{*}
$$

where $\left\langle l_{i p} l_{j q} l_{k r} l_{l s}\right\rangle$ - sign of averaging over the ensemble of random fiber orientations, $c_{p k r s}^{*}$ - components of the elasticity tensor of a unidirectionally reinforced composite with the same volume reinforcement coefficient, $l_{i p}-$ cosines of angles between the main axes of symmetry of the spatially reinforced composite and the axes of the local coordinate system associated with the direction of reinforcement of the elementary volume.

Because of the assumptions about the ergodicity of the model, the averaging volume of the composite and the ensemble of random orientations coincide, which allows for the calculation of elastic macroscopic characteristics of the spatial distribution of the fibers is described using a density distribution of spherical angles specifying the position of a randomly oriented fiber axes associated with the composite. Further, suppose that isotropic or transversally isotropic fibers are distributed in an isotropic matrix in such a way that three orthogonal planes of elastic symmetry pass through each point of the material, i.e. the composite is orthotropic and is characterized by nine independent elastic constants

We obtain a system of algebraic equations to determine the nine independent coefficients of elasticity of the orthotropic composite. In matrix notation, its solution is in the form of:

$$
\begin{aligned}
& c_{11}=c_{11}^{*}-\left(2 c_{1}^{*}+4 c_{2}^{*}\right) \Delta_{1}+c_{3}^{*} \Delta_{4}, \\
& c_{22}=c_{11}^{*}-\left(2 c_{1}^{*}+4 c_{2}^{*}\right) \Delta_{2}+c_{3}^{*} \Delta_{5}, \\
& c_{33}=c_{11}^{*}-\left(2 c_{1}^{*}+4 c_{2}^{*}\right) \Delta_{3}+c_{3}^{*} \Delta_{6}, \\
& c_{23}=c_{13}^{*}+\left(c_{1}^{*}-c_{3}^{*}\right) \Delta_{1}+\frac{c_{3}^{*}}{2}\left(1+\Delta_{4}-\Delta_{5}-\Delta_{6}\right), \\
& c_{13}=c_{13}^{*}+\left(c_{1}^{*}-c_{3}^{*}\right) \Delta_{2}+\frac{c_{3}^{*}}{2}\left(1+\Delta_{5}-\Delta_{6}-\Delta_{4}\right), \\
& c_{12}=c_{13}^{*}+\left(c_{1}^{*}-c_{3}^{*}\right) \Delta_{3}+\frac{c_{3}^{*}}{2}\left(1+\Delta_{6}-\Delta_{4}-\Delta_{5}\right), \\
& c_{44}=c_{44}^{*}+\left(c_{2}^{*}-c_{3}^{*}\right) \Delta_{1}+\frac{c_{3}^{*}}{2}\left(1+\Delta_{4}-\Delta_{5}-\Delta_{6}\right), \\
& c_{55}=c_{44}^{*}+\left(c_{2}^{*}-c_{3}^{*}\right) \Delta_{2}+\frac{c_{3}^{*}}{2}\left(1+\Delta_{5}-\Delta_{6}-\Delta_{4}\right), \\
& c_{66}=c_{44}^{*}+\left(c_{2}^{*}-c_{3}^{*}\right) \Delta_{3}+\frac{c_{3}^{*}}{2}\left(1+\Delta_{6}-\Delta_{4}-\Delta_{5}\right),
\end{aligned}
$$

where the following designations for material constants are introduced: $c_{1}^{*}=c_{12}^{*}-c_{13}^{*}, \quad c_{2}^{*}=c_{66}^{*}-c_{44}^{*}$, $c_{3}^{*}=c_{11}^{*}+c_{33}^{*}-2 c_{13}^{*}-4 c_{44}^{*}$; textural parameters $\Delta_{i}$, uniquely determining the elastic properties of the material. The average values of the 2-nd and 4-th degrees of the cosines of the angles between the fiber axis and the laboratory coordinate system were chosen as texture parameters.

The resulting expression allows us to establish the elastic characteristics of the spatially reinforced composite on the elastic characteristics of unidirectional reinforced material and well-defined texture parameters for any distribution of fibers in the material under the restriction that the material is orthotropic.

Then we define the characteristics of the multiphase material by the formula: $\mathrm{c}^{*}=\sum_{i=1}^{n} f_{i} c_{i}^{*}$, where $f_{i}$ - the bulk content of the material of the i-type in the general composite, and $f_{i}=v_{i} K$; coefficient $\mathrm{K}$ determined from the condition $\sum_{k=1}^{n} f_{i}=1$. 


\section{CALCULATION OF ELASTIC CHARACTERISTICS ON THE EXAMPLE OF ALUMINUM ALLOY}

For example, it is possible to calculate the elastic characteristics of a composite material that has practical application in the technique based on an aluminum alloy reinforced with fiber - borsik and additionally reinforced with high-strength steel wire laid perpendicular to the boron fiber. The introduction of steel wire allows increasing the strength of the material in the transverse direction by $1.5-2.5$ times, without requiring significant changes in the technological process of its manufacture compared to the composition of aluminum -boron.

We denote the characteristics of the matrix, boron fibers and steel wire, respectively $-c_{i j k l}^{m}, c_{i j k l}^{(1)}, c_{i j k l}^{(2)}$.

Volume content: $v_{m}=0,5, v_{1}=0,3, v_{2}=0,2$.

Let the fibers of the first type is directed along the axis $O x_{1}$, and the fibers of the second type is directed in a perpendicular position along the axis $\mathrm{Ox}_{2}$.

Calculation of elastic characteristics will be carried out in several stages. In the first stage determined the effective properties of the composite $\mathrm{Al}-\mathrm{B} / \mathrm{SiC}$ unidirectional-reinforced system of fibers along the axis $O x_{1}-\mathrm{c}_{1}^{*}$, and composite Al-AFC77 unidirectional reinforced steel wire along the axis $O x_{2}-c_{2}^{*}$. The effective elastic characteristics of a unidirectionally reinforced composite can be calculated from the formulas [1] . Next, to determine the nine independent coefficients of elasticity of the orthotropic composite, we put in formulas (1) for $\mathrm{c}_{1}^{*}$ $\Delta_{1}=\Delta_{4}=1, \Delta_{2}=\Delta_{3}=\Delta_{5}=\Delta_{6}=0 ; c_{2}^{*}: \Delta_{2}=\Delta_{5}=1, \Delta_{1}=\Delta_{3}=\Delta_{4}=\Delta_{6}=0$.

It is also necessary to determine the relative volume concentration of inclusions and matrix for each material under consideration: $p_{i}=\frac{v_{i}}{f_{i}}, p_{m}=\frac{f_{i}-v_{i}}{f_{i}}$, where $f_{i}$ - the bulk content of the material type 1 or 2 in the total composite, $f_{i}=v_{i} K$, and coefficient $\mathrm{K}$ determined from condition $f_{1}+f_{2}=1$.

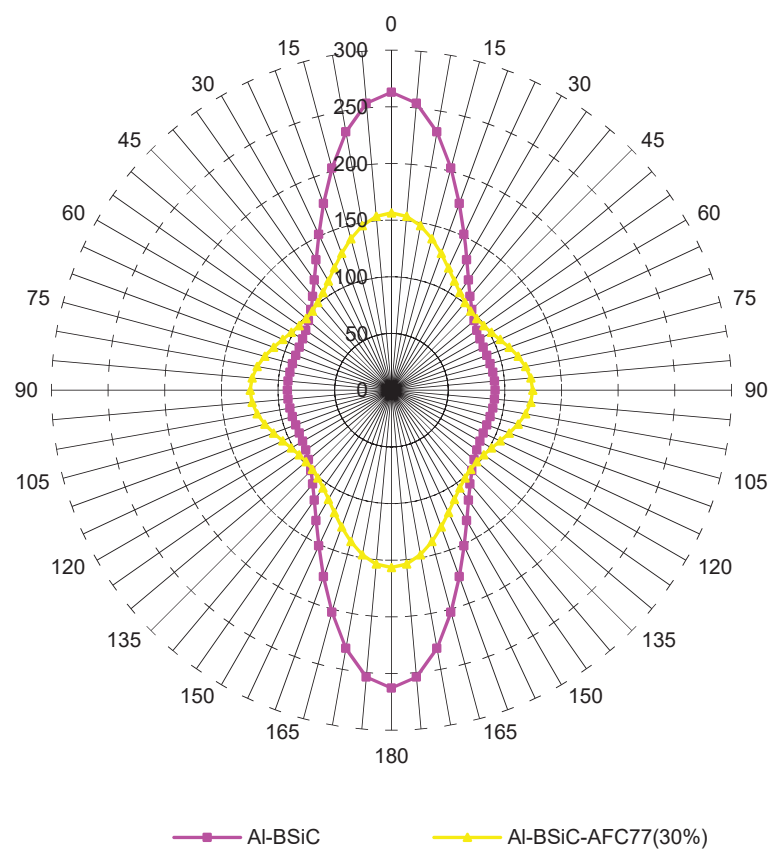

FIGURE 1. Anisotropy of young's modulus.

In the second stage, given that the composite material in question consists of a matrix and inclusions of two types, we determine the elastic characteristics of such a material from the expression: $c^{*}=\mathrm{c}_{1}^{*} f_{1}+\mathrm{c}_{2}^{*} f_{2}$. 
Having calculated the elastic modulus by the proposed method, it is possible to investigate the anisotropy of young's modulus by substituting them into the ratio. The results of calculations by the proposed method are presented in Fig. 1.

Also there is a comparison with a composite material based on aluminum reinforced with borsik fibers: Al-B$\mathrm{SiC}$. The introduction of steel wire allows increasing the strength properties of the material, while changing slightly the stiffness characteristics

\section{CONCLUSION}

The obtained expression for the elastic characteristics of a spatially reinforced multiphase composite material allows, by varying the elastic characteristics of the phase components, the volume concentrations and the distribution of fibers in space, to obtain a material with the required level of properties.

\section{REFERENCES}

[1] E.M. Romanovskaia, E.A. Mityushov, S.A. Berestova, N.D. Romanovskaia, "Forecasting of the elastic properties of fibrous materials", AIP Conference Proceedings 2116, 430007 (2019); https://doi.org/10.1063/1.5114444. 